\title{
In vivo testing of novel vaccine prototypes against Actinobacillus pleuropneumoniae
}

\author{
Fabio Antenucci', Cyrielle Fougeroux ${ }^{2}$, Alannah Deeney ${ }^{3}$, Cathrine Ørskov ${ }^{4}$, Andrew Rycroft ${ }^{3}$, \\ Peter Johannes Holst ${ }^{2}$ and Anders Miki Bojesen ${ }^{1 *}$ (D)
}

\begin{abstract}
Actinobacillus pleuropneumoniae (A. pleuropneumoniae) is a Gram-negative bacterium that represents the main cause of porcine pleuropneumonia in pigs, causing significant economic losses to the livestock industry worldwide. A. pleuropneumoniae, as the majority of Gram-negative bacteria, excrete vesicles from its outer membrane (OM), accordingly defined as outer membrane vesicles (OMVs). Thanks to their antigenic similarity to the OM, OMVs have emerged as a promising tool in vaccinology. In this study we describe the in vivo testing of several vaccine prototypes for the prevention of infection by all known A. pleuropneumoniae serotypes. Previously identified vaccine candidates, the recombinant proteins ApfA and VacJ, administered individually or in various combinations with the OMVs, were employed as vaccination strategies. Our data show that the addition of the OMVs in the vaccine formulations significantly increased the specific lgG titer against both ApfA and VacJ in the immunized animals, confirming the previously postulated potential of the OMVs as adjuvant. Unfortunately, the antibody response raised did not translate into an effective protection against A. pleuropneumoniae infection, as none of the immunized groups following challenge showed a significantly lower degree of lesions than the controls. Interestingly, quite the opposite was true, as the animals with the highest lgG titers were also the ones bearing the most extensive lesions in their lungs. These results shed new light on A. pleuropneumoniae pathogenicity, suggesting that antibody-mediated cytotoxicity from the host immune response may play a central role in the development of the lesions typically associated with A. pleuropneumoniae infections.
\end{abstract}

\section{Introduction}

Actinobacillus pleuropneumoniae (A. pleuropneumoniae) is a Gram-negative bacterium responsible for a serious respiratory disease affecting pigs and a cause of large economic losses in the pig industry $[1,2]$. A. pleuropneumoniae is transmitted from pig to pig by aerosols or direct contact and causes clinical signs such as vomiting, diarrhea, respiratory distress and bloody discharge from the mouth and nose, which may be lethal [3]. A total of 16 different $A$. pleuropneumoniae serotypes have

\footnotetext{
*Correspondence: miki@sund.ku.dk

${ }^{1}$ Department of Veterinary and Animal Sciences, University

of Copenhagen, Stigbøjlen 4, 1870 Frb. C., 1-20, Building: 301,

Copenhagen, Denmark

Full list of author information is available at the end of the article
}

been reported worldwide, classified according to capsular antigens $[4,5]$. Several serotypes contain strains that can cause severe symptoms, which makes it challenging to develop a broadly protective vaccine. Currently available vaccines against App can be divided in two categories: (i) vaccines based on inactivated wholecell bacterins; (ii) vaccines based on Apx toxins, a set of pore-forming cytolysins (Apx I-IV) playing a central role in App pathogenesis [3, 6]. Bacterin-based vaccines have been shown to offer limited protection against infections by strains other than the ones used for vaccination $[7,8]$. While vaccines based on inactivated Apx toxins (toxoids) are effective in reducing the morbidity associated with infection [9-12] yet unable to prevent colonisation of the lungs, their use pose a potential threat inducing infection by asymptomatic carriers $[7,11,13]$. Thus, it would 
be highly desirable to develop a novel vaccine offering protection against App colonisation and morbidity in a cross-serotype manner.

The poor specificity of the detection methods for $A$. pleuropneumoniae and the rapid progression of the infection frequently prompt widespread application of antibiotic treatment on the mere suspicion of an infection [14]. This contributes to the overuse of antibiotics, which promotes selection of resistant bacteria and may indirectly lead to the spread of antibiotic resistance genes through the food chain $[15,16]$. Accordingly, an effective vaccine against $A$. pleuropneumoniae, which protects across the 16 serovars is urgently needed.

The pathogenesis of porcine pleuropneumonia is complex and currently divided in different stages: colonization, resistance to clearance and damage to host tissues (direct and indirect) $[3,17,18]$. Colonization is usually the fist step in the establishment of a bacterial infection, and several $A$. pleuropneumoniae virulence factors have been shown to be involved in adhesion to the host tissues and expressed in vivo $[19,20]$. A. pleuropneumoniae cells also show the ability to avoid clearance by both innate and adaptive immune responses thanks to mechanisms such as antiphagocytic activity and antibody degradation $[3,6,21]$. Finally, the lesions typically associated with porcine pleuropneumonia are mostly caused by a combination of toxins and proteases secreted by the pathogen and inflammatory mediators released by activated phagocytes $[3,22,23]$.

As previously mentioned, cross-serotype prevention of porcine pleuropneumoniae remains elusive, and successful control of the infection with vaccination may require more in-depth knowledge of the pathogenesis of the disease. It is thus important to clarify how the establishment of the infection affects the course of the disease and if, as suggested; acute lung damage developed in the first days is the defining features for the severity of the disease [24]. Moreover it would be informative to understand if the infection can be controlled on a systemic level or mostly on a local level, thus determining the most efficient route of immunization.

In this study we describe the adoption of three different vaccination strategies for the prevention of porcine pleuropneumonia and lung colonization by $A$. pleuropneumoniae. Different strategies were selected in order to offer to the host immune system the widest possible range of immunogens and alternative administration routes. The first strategy involved the utilization of previously selected cross-serotype immunogens [25], expressed as recombinant proteins. Briefly, the conserved immunogens were selected in silico on the basis of exposition and accessibility of the proteins on the bacterial outer membrane $(\mathrm{OM})$, as well as gene conservation among serovars, as described in [25]. From these studies we selected the proteins Apfa and VacJ, a pilin and an OM lipoprotein, respectively, whose role in A. pleuropneumoniae virulence previously has been described [11, 26, 27]. The selection of ApfA and VacJ as immunogens offered the potential of targeting $A$. pleuropneumoniae cells adhesion, OM integrity and resistance to clearance by the host immune system.

The second strategy involved the utilization of the antigenic outer membrane vesicles (OMVs) [28]. OMVs possess a variety of biological functions, and most importantly usually exhibit an antigenic pattern similar to the one found on the bacterial OM $[29,30]$. Accordingly, $A$. pleuropneumoniae OMVs have shown promising results in terms of immunogenicity, offering the prospect of providing a range of conserved antigens [31]. Furthermore, thanks to their non-live nature, OMVs can be employed in vaccine formulations without incurring in the usual risks associated with live vaccines [29]. Notably, both autologous and heterologous OMVs have been reported to function as an adjuvant for co-administered recombinant antigens [32-34], but more recently some controversy has emerged regarding the possibility of combining OMVs with other immunogens [35]. To verify the potential of OMVs as adjuvants, we decided to combine OMVs and recombinant proteins ApfA and VacJ as a third strategy of immunization.

One of the technical challenges during an infection trial is to ensure the thoroughly and equivalent dissemination of infectious particles through the groups of animals. As previously mentioned, one of the main transmission routes of $A$. pleuropneumoniae infections is by aerosol [36, 37]. To simulate as closely as possible natural transmission, we selected an aerosol chamber transmission model as dissemination procedure for our in vivo challenge. This system is ideally suited for a respiratory pathogen such $A$. pleuropneumoniae, and has already been tested successfully in several studies [38-40].

\section{Materials and methods Animal model}

A total of $5510-\mathrm{kg}$ piglets (Landrace-Yorkshire-Duroc crossbred) were purchased from a commercial breeder, which was part of the Danish Specific-Pathogen-Free (SPF) breeding system (code red), which is the highest health level in the system. Briefly, code red SPF herds are tested monthly for App clinical signs and by a serum sample ELISA aiming at serotypes $1-10$ and 12, respectively. Upon arrival the pigs were housed in the animal facilities at the Department of Veterinary and Animal Sciences, (Frederiksberg, Copenhagen, Denmark). Piglets were ear-tagged with a unique identification number and randomly distributed in four different rooms that could 
be further separated in three smaller pens, after vaccination. Experiments were started after allowing the pigs to acclimatize for a week. Daily care was provided by animal caretakers blinded to treatment groups. Experiments were approved by the Danish national animal experiments inspectorate (Dyreforsøgstilsynet), as stipulated in license number 2014-15-0201-00019. Pigs were checked for presence of $A$. pleuropneumoniae by plating nose swaps on MHF plates [Mueller-Hinton agar $+5 \%$ horse blood $+20 \mathrm{mg} / \mathrm{L}$ ß-nicotinamide dinucleotide (NAD); BioMérieux], before initiation of the experiment.

\section{Immunogen production}

Recombinant proteins ApfA and VacJ, and OMVs were produced and isolated as described in [25]. Briefly, apfA and vacJ genes from A. pleuropneumoniae L20 (serotype $5 \mathrm{~b})$ were cloned and expressed in competent Escherichia coli (E. coli) BL21 (DE3) cells, and the resulting ApfA and
VacJ proteins isolated by size exclusion chromatography as described in [41]. OMVs were produced from A. pleuropneumoniae MIDG2331 (serotype 8) cultures and isolated by hydrostatic filtration, as described in [25].

\section{Immunization}

The immunization and challenge procedure is summarized in Figure 1. After 1-week of acclimatization pigs were vaccinated intra-muscularly and intranasally either with OMVs, recombinant proteins or a combination of OMVs and recombinant proteins (Table 1). Intramuscular (IM) immunizations were done by injecting $1 \mathrm{~mL}$ of the vaccine into the muscle on the dorsal side of the neck. Intranasal (IN) immunizations were made with $1 \mathrm{~mL}$ volume of the vaccine instilled in one of the nostrils with a plastic Pasteur pipette (Deltalab). Group 1 (control) was administered $1 \mathrm{~mL}$ phosphate buffered saline (PBS). Groups 2, 3, 5, 6 and 7 were vaccinated with $30 \mu \mathrm{g}$

\begin{tabular}{|c|c|c|c|c|c|}
\hline \multicolumn{2}{|c|}{$\begin{array}{l}\text { WEEK 0 } \\
\text { Arrival of the } \\
\text { pigs }\end{array}$} & $\begin{array}{c}\text { WEEK } 1 \\
1^{\text {st }} \\
\text { immunization }\end{array}$ & $\begin{array}{c}\text { WEEK } 5 \\
2^{\text {nd }} \\
\text { immunization }\end{array}$ & $\begin{array}{l}\text { WEEK } 9 \\
\text { APP } \\
\text { challenge }\end{array}$ & $\begin{array}{l}\text { WEEK } 10 \\
\text { necropsy }\end{array}$ \\
\hline$\frac{\text { Group } 1}{4 \text { pigs }}$ & & PBS $(\mathrm{IM}+\mathrm{IN})$ & PBS $(\mathrm{IM}+\mathrm{IN})$ & \multirow{7}{*}{$\begin{array}{l}10^{6} \mathrm{CFU} / \mathrm{mL} \text { in } \\
\text { aerosol chamber }\end{array}$} & \\
\hline$\frac{\text { Group } 2}{4 \text { pigs }}$ & & $\begin{array}{l}\text { VacJ protein }(\mathrm{IM}+\mathrm{IN}) \\
(30 \mu \mathrm{g} / \mathrm{mL})\end{array}$ & $\begin{array}{l}\text { VacJ protein (IM+IN) } \\
(30 \mu \mathrm{g} / \mathrm{mL})\end{array}$ & & \\
\hline$\frac{\text { Group } 3}{4 \text { pigs }}$ & & $\begin{array}{l}\text { ApfA protein (IM+IN) } \\
(30 \mu \mathrm{g} / \mathrm{mL})\end{array}$ & $\begin{array}{l}\text { ApfA protein (IM+IN) } \\
(30 \mu \mathrm{g} / \mathrm{mL})\end{array}$ & & \\
\hline$\frac{\text { Group } 4}{4 \text { pigs }}$ & & $\begin{array}{l}\text { OMVs (IM+IN) } \\
\left(1,62.10^{10} \text { vesicles }\right)\end{array}$ & $\begin{array}{l}\text { OMVs (IM+IN) } \\
\left(1,62.10^{10} \text { vesicles }\right)\end{array}$ & & \\
\hline$\frac{\text { Group } \mathbf{5}}{4 \text { pigs }}$ & & $\begin{array}{l}\text { OMVs }+ \text { ApfA protein }(\mathrm{IM}+\mathrm{IN}) \\
\left(1,62.10^{10} \text { vesicles }\right)+(30 \mu \mathrm{g} / \mathrm{mL})\end{array}$ & $\begin{array}{l}\text { OMVs +ApfA protein }(\mathrm{IM}+\mathrm{IN}) \\
\left(1,62.10^{10} \text { vesicles }\right)+(30 \mu \mathrm{g} / \mathrm{mL})\end{array}$ & & \\
\hline$\frac{\text { Group } 6}{4 \text { pigs }}$ & & $\begin{array}{c}\text { OMVs +VacJ protein }(\mathrm{IM}+\mathrm{IN}) \\
\left(1,62.10^{10} \text { vesicles }\right)+(30 \mu \mathrm{g} / \mathrm{mL})\end{array}$ & $\begin{array}{l}\text { OMVs +VacJ protein }(\mathrm{IM}+\mathrm{IN}) \\
\left(1,62.10^{10} \text { vesicles }\right)+(30 \mu \mathrm{g} / \mathrm{mL})\end{array}$ & & \\
\hline$\frac{\text { Group } 7}{5 \text { pigs }}$ & & $\begin{array}{l}\text { OMVs }+ \text { VacJ }+ \text { ApfA protein }(\mathrm{IM}+\mathrm{IN}) \\
62.10^{10} \text { vesicles }+((30 \mu \mathrm{g} / \mathrm{mL})+(30 \mu \mathrm{g} / \mathrm{mL})\end{array}$ & $\begin{array}{c}\text { OMVs +VacJ +ApfA protein }(\mathrm{IM}+\mathrm{IN}) \\
\left(1,62.10^{10} \text { vesicles }\right)+(30 \mu \mathrm{g} / \mathrm{mL})+(30 \mu \mathrm{g} / \\
\mathrm{mL})\end{array}$ & & \\
\hline
\end{tabular}

Figure 1 Immunization and challenge regimen. Immunizations were carried out intra-nasally with a Pasteur pipette and intra-muscularly by injection. Animals were immunized with either a single component or a combination of the following: $30 \mu \mathrm{g} / \mathrm{mL}$ protein (Apfa and/or VacJ), $1.62 \times 10^{10}$ vesicles $/ \mathrm{mL}$ OMV. All animals were re-immunized (boosted) 4 weeks after the first immunization. Finally, all pigs were challenged 8 weeks after the first immunization with live A. pleuropneumoniae HK361 via an aerosol chamber. A week after challenge, pigs were euthanized and subjected to necropsy.

Table 1 Immunization groups

\begin{tabular}{|c|c|c|c|}
\hline Group & Number of pigs & Vaccination & Route of immunization \\
\hline 1 & 4 & PBS & $I M+I N$ \\
\hline 2 & 4 & VacJ protein & $\mathrm{IM}+\mathrm{IN}$ \\
\hline 3 & 4 & ApfA protein & $I M+I N$ \\
\hline 4 & 4 & A. pleuropneumoniae OMV & $\mathrm{IM}+\mathrm{IN}$ \\
\hline 5 & 4 & A. pleuropneumoniae OMV + VacJ protein & $\mathrm{IM}+\mathrm{IN}$ \\
\hline 6 & 4 & A. pleuropneumoniae OMV + ApfA protein & $I M+I N$ \\
\hline 7 & 5 & A. pleuropneumoniae OMV + VacJ protein + ApfA protein & $\mid M+I N$ \\
\hline
\end{tabular}


(per protein) of recombinant protein and groups 4-7 were vaccinated with $1.62 \times 10^{10} \mathrm{OMV}$. The OMV dosage was set not to exceed tolerable lipopolysaccharide levels. Groups from 1 to 7 were boosted 4 weeks after the first immunization with a similar vaccine and dose as the first vaccination. Blood was drawn using the vacutainer system with clot activator (Vacutainer) from the jugular vein on day 0 , week 2 , week 4 , week 6 and week 8 after the first vaccination and later, on the day of the A. pleuropneumoniae challenge, and three and 7 days post challenge, respectively.

\section{Challenge}

All pigs were challenged with live A. pleuropneumoniae HK361 cells [serotype 2; National Collection of Type Cultures (NCTC) 10976], 8 weeks after the first vaccination. A. pleuropneumoniae bacteria from an overnight BHI agar culture were incubated in brain-heart infusion (BHI) media supplemented with $5 \mu \mathrm{g} / \mathrm{mL}$ NAD (Sigma Aldrich). When the optical density $600 \mathrm{~nm}$ $\left(\mathrm{OD}_{600 \mathrm{~nm}}\right)$ reached 1.5 [corresponding to approximately $2.10^{9}$ colony-forming units (CFU)/mL according to pilot experiments], the culture was diluted 1:2000 in $30 \mathrm{~mL}$ of HEPES saline (10 mM HEPES; $150 \mathrm{mM} \mathrm{NaCl}, 3 \mathrm{mM} \mathrm{KCl}$, $1 \mathrm{mM} \mathrm{CaCl}$ and $1 \mathrm{mM} \mathrm{MgCl}$ ) to obtain a concentration of $10^{6} \mathrm{CFU} / \mathrm{mL}$. The diluted solution $\left(10^{-1}\right)$ was then administered as an aerosol using a NE-U17 OMRON Ultrasonic nebuliser (OMRON Healthcare) and an aerosol chamber where four to five pigs at a time were left for $10 \mathrm{~min}$ in a A. pleuropneumoniae aerosol. Each chamber session was ended by a period of five min venting, to allow clearing of aerosolised A. pleuropneumoniae by passing all air through a HEPA filter before releasing the pigs from the chamber. Pigs were challenged over 2 days, and overall two batches of $A$. pleuropneumoniae were produced and each batch was used for two to three groups of four to five pigs. The initial (pre challenge) $A$. pleuropneumoniae solution $\left(10^{-1}\right)$ and the leftover solution (post challenge) were plated and incubated on MHF plates in duplicates, for recollection and CFU counting the next day.

\section{Animal welfare}

Clinical signs of the animals were monitored every $3-4 \mathrm{~h}$ during the period between challenge and euthanasia. Animals showing signs of distress or a body temperature $>40{ }^{\circ} \mathrm{C}$ were administered IM injections of butorphanol tartrate (Torbugesic, Zoetis), dosed according to weight and previous treatment.

\section{Post-mortem examination}

Pigs were sacrificed 1 week after the A. pleuropneumoniae challenge; lungs were excised from the thorax.
Samples from lesions and healthy areas of the lungs were excised and placed in 10\% formalin for histological analysis. The pleura and macroscopic lesions of the lungs were assessed systematically using a previously reported scoring system (Figure 2) [42]. Briefly, each of the seven lobes of each lung was assigned a score [0-5] depending on the number and size of lesions. From that the total score of the whole lung was calculated. The statistical significance of the data was assessed using Ordinary One-way ANOVA, Brown-Forsythe, Bartlett's $(P<0.05)$ and Dunnett's multiple comparisons (alpha $=0.05)$ tests. Correlation between data sets was assessed by Pearson $r$ two-tailed test (alpha $=0.05)$. After scoring, each lung was weighed and added 1/1 (weight/vol) $0.9 \%$ sterile saline solution (Thermo Fisher Scientific) and blended to obtain a homogenized mixture, which was serially diluted tenfold between $1: 10^{-1}$ and $1: 10^{-7} .10 \mu \mathrm{L}$ of each dilution was then plated on MHF plates in duplicates. After overnight $(\mathrm{ON})$ incubation, $\mathrm{CFU}$ counts were adjusted

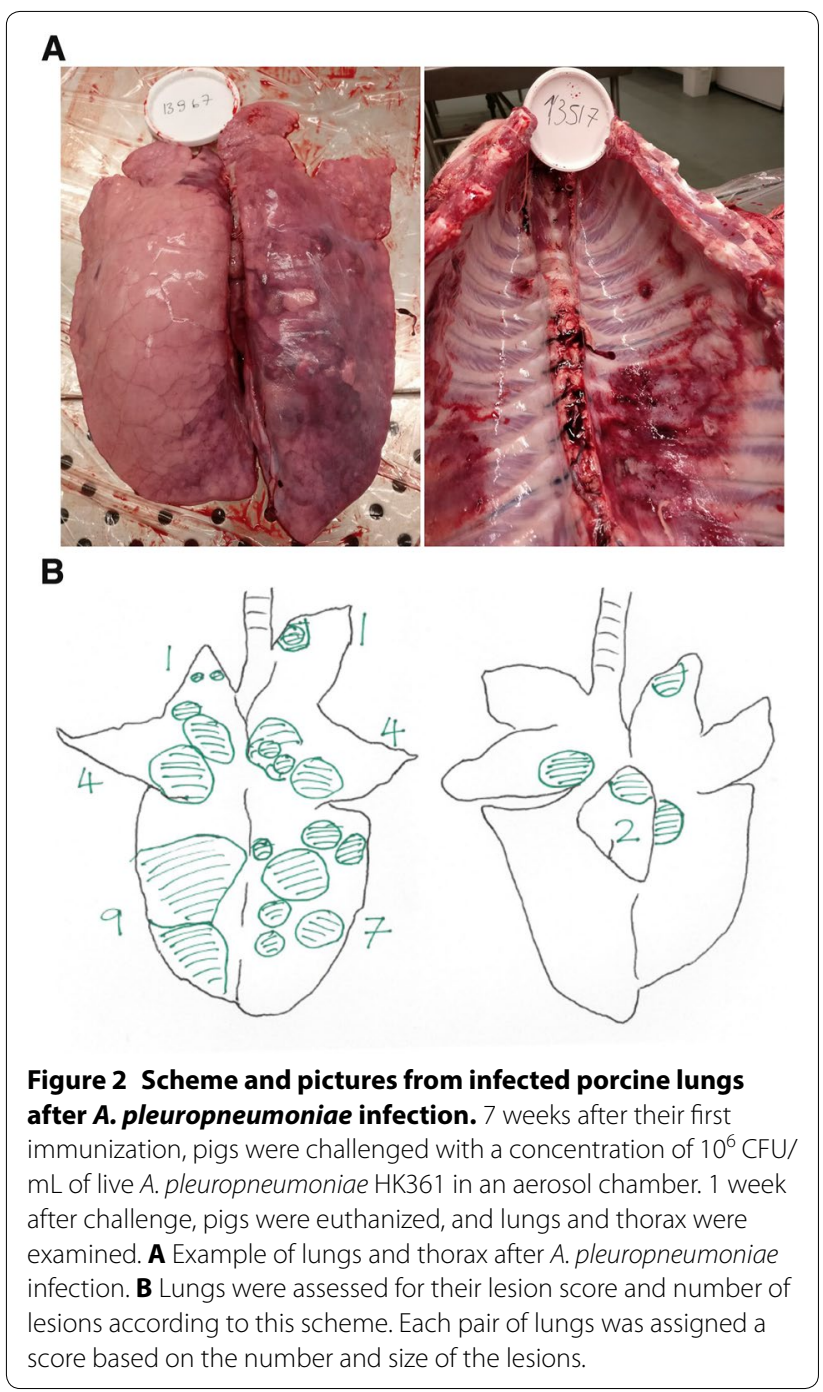


in order to reflect the total amount of bacteria contained per gram of lung (CFU/g).

\section{Antibody titration}

Sera were isolated from blood samples by allowing the samples to clot. Nunc Maxisorp flat-bottom 96-wells plates (Thermo Fisher Scientific) were coated with $2 \mu \mathrm{g} /$ mL ApfA or Vacj proteins, or $3.25 \times 10^{8}$ OMVs in PBS, respectively. Sera from animals in the same group were pooled and added to the wells in three-fold dilutions between 1:500 and 1:1 093500 (ApfA, VacJ) or 1:1000 and 1:2 187000 (OMVs). Antibodies specifically recognizing ApfA, VacJ and OMVs were detected with horseradish peroxidase (HRP)-conjugated polyclonal rabbit anti-pig IgG (Thermo Fisher Scientific). Wells were revealed using TMB plus (Kem-En-Tec Diagnostics). Optical density was measured at $450 \mathrm{~nm}$ using an enzyme-linked immunosorbent assay (ELISA) plate reader (VersaMax Molecular Devices).

\section{Results}

Inocula analysis

Actinobacillus pleuropneumoniae CFU counts from the inocula are summarized in Table 2 . No statistically different CFU count was found between different batches, groups or days of challenge resulting in an expected similar inhaled dose for all pigs.

\section{Histopathological analysis of the lungs}

Lesion scores of the lungs are shown in Figure 3. Affected lungs presented lesions from subacute to acute, with signs of multifocal necrosis, fibrinous exudation and purulent inflammation. In some cases the extensive fibrinous exudation to the pleural surface had led to extensive adhesive pleuritis (Figure 4). Some of the most severely affected lungs were partially or completely collapsed, suggesting loss of function (Figure 4). Statistical analysis showed no significant difference between mean lesion scores and standard deviations of each individual group (Figure 3), indicating that none of the vaccine formulations administered were able to successfully prevent $A$. pleuropneumoniae associated colonization and lesions. Instead, statistical analysis by Pearson $\mathrm{r}$ two-tailed test showed a correlation between the number of $A$. pleuropneumoniae

\section{Table 2 Inocula analysis}

\begin{tabular}{llll}
\hline Batch & $\begin{array}{l}\text { Administered } \\
\text { to group }\end{array}$ & $\begin{array}{l}\text { CFU/mL (pre-chal- } \\
\text { lenge) }\end{array}$ & $\begin{array}{l}\mathrm{CFU} / \mathrm{mL} \text { (post- } \\
\text { challenge) }\end{array}$ \\
\hline 1 & $1,2,3,4$ & $2 \times 10^{6}$ & $3.25 \times 10^{6}$ \\
2 & $5,6,7$ & $1.46 \times 10^{6}$ & $1.85 \times 10^{6}$ \\
\hline
\end{tabular}

Serial dilutions from the inocula before and after challenge were plated on MHF plates. CFU counts are expressed as CFU/mL.

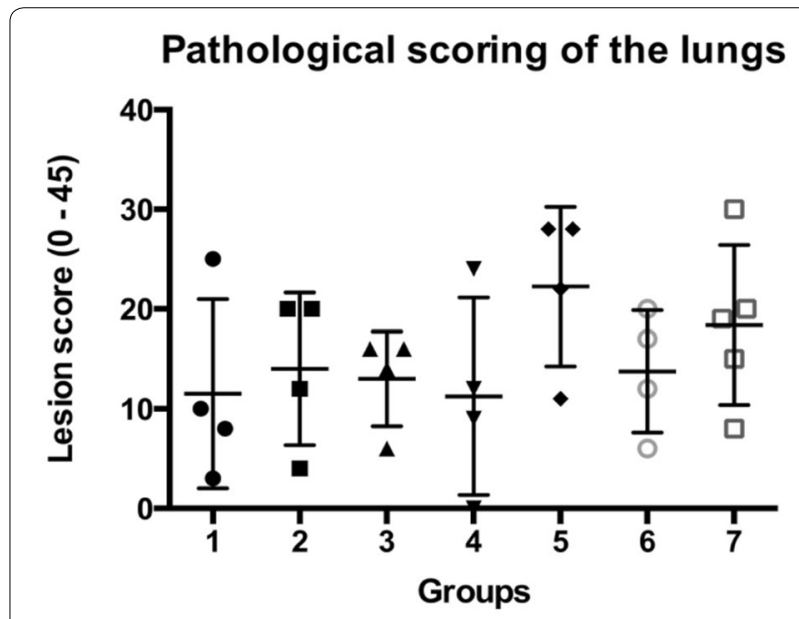

Figure 3 Lesion scores of the lungs. Lungs were scored for lesions as previously described [42]. Mean and standard deviation values are graphically reported in figure. Statistical analysis by Ordinary One-way ANOVA, Brown-Forsythe, Bartlett's and Dunnett's multiple comparisons tests showed no significant difference between mean scores and standard deviations of each individual group. 1: PBS; 2:VacJ; 3: ApfA; 4: OMVs; 5: VacJ + OMVs; 6: ApfA + OMVs; 7 : $\mathrm{VacJ}+\mathrm{ApfA}+\mathrm{OMVs}$

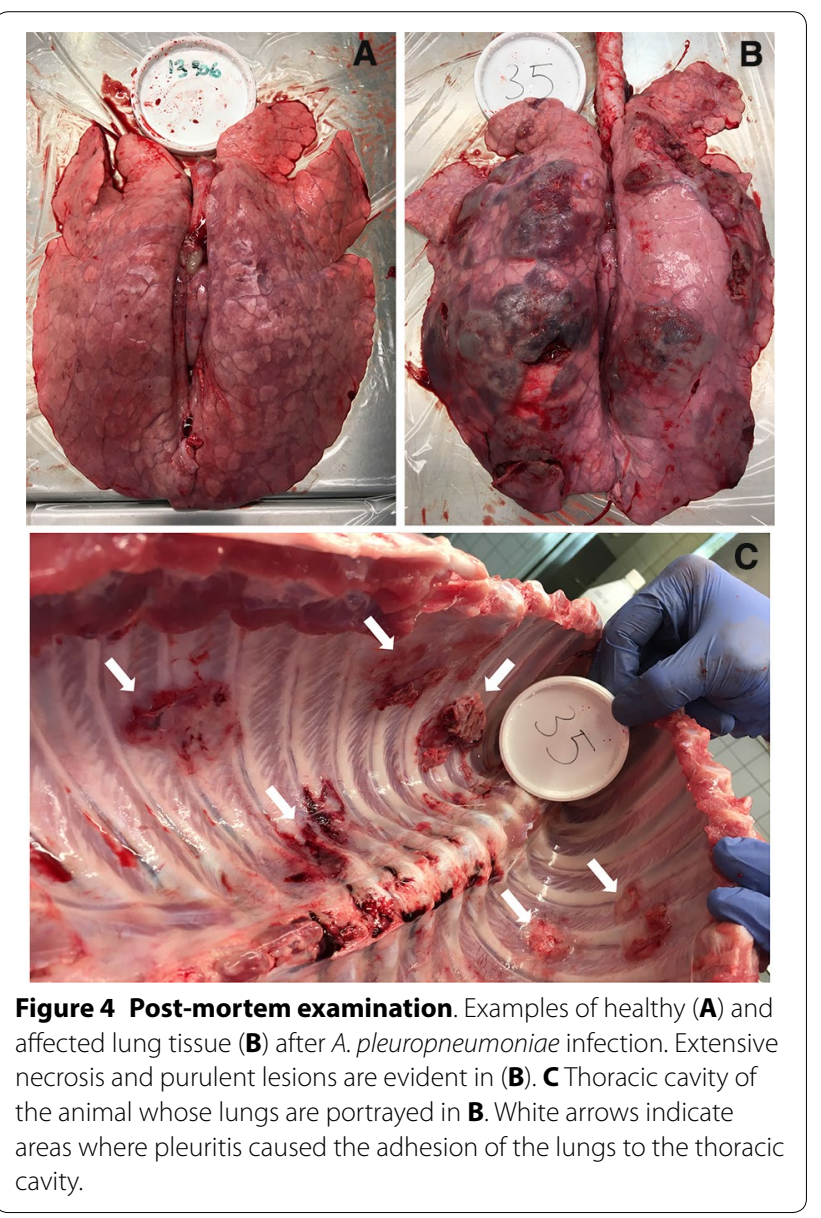


CFU present in the lungs and lesion scores $(P<0.0001)$ (Figure 5), validating the pathological evaluation perfor med.

Histological analysis of the lesions revealed intense lymphocytic infiltration, with loss of structural integrity and occlusion of the alveoli (Figure 6). No difference could be observed between individual groups, with sections of the lungs similarly affected exhibiting comparable histological characteristics.

\section{Characterization of $\mathrm{IgG}$ response to the immunogens}

IgG titers from pig sera are shown in Figure 7. The sera retrieved from animals belonging to the same group were pooled together for immunological analysis. As such, the serological data and the resulting conclusions presented in this study are to be considered representative of trends per group rather than per individual animal.

Immunization with ApfA and VacJ proteins alone did not produce an appreciable increase in specific IgG antibodies against the proteins (Figure 7), suggesting the ineffectiveness of immunizing with these proteins individually. Furthermore, immunization with ApfA induced an IgG response against VacJ in the sera of vaccinated animals, suggesting that ApfA may interfere with the development of immunity against co-administered immunogens, as reported before [43]. Interestingly, the animals in the control group presented a rather high titer against VacJ after challenge, pointing to the in vivo immunogenicity of this protein (Figure 7). As expected given their multi-antigenic nature, the overall antibody response to the OMVs was conspicuous in all groups, with the highest titers reached when the OMVs were administered alone (Figure 7). Administration

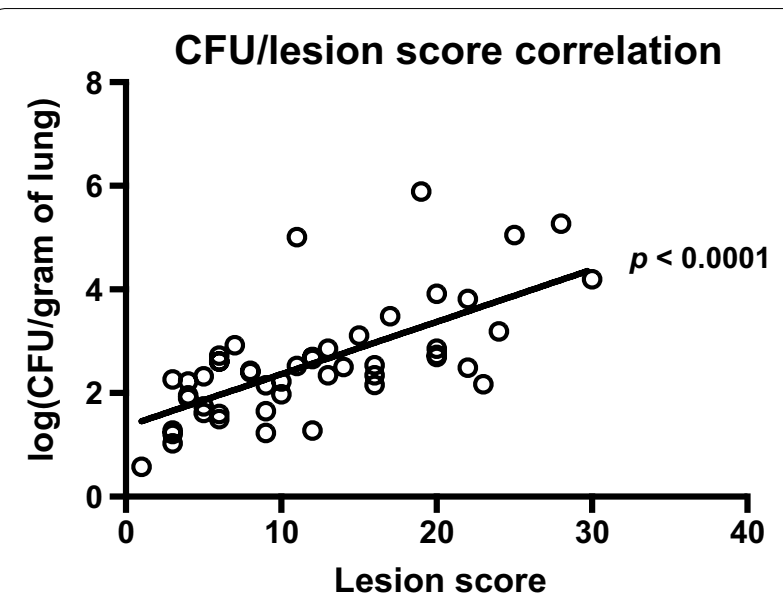

Figure 5 CFU/Lesion score correlation. The number of A. pleuropneumoniae CFU retrieved from the lungs after euthanasia was plotted against the corresponding lesion score for each individual animal. Statistical analysis by Pearson two-tailed $r$ test showed a significant correlation between number of CFU and lesion score $(P<0.0001)$. in combination with either of the other immunogens decreased the response to the OMVs, particularly before challenge, increasing on the other hand the response to the other immunogens (Figure 7). This was especially true in the case of Vac), were groups that received both VacJ and OMVs showed steadily increasing and overall high IgG titers, while groups that received only VacJ showed a decrease in titer, even after challenge (Figure 7). Furthermore, statistical analysis by Pearson $r$ two-tailed test showed a positive correlation between IgG titers elicited against the ApfA and VacJ immunogens and lesion scores (ApfA $P=0.04$; VacJ $P=0.03$ ), suggesting a major negative role of the immune system in $A$. pleuropneumoniae pathogenesis (Figure 8). No correlation was observed between IgG response to the OMVs and lesion scores.

\section{Discussion}

Effective prevention of $A$. pleuropneumoniae outbreaks has remained elusive for decades, with available vaccines unable to confer cross-serotype protection or prevent lung colonization by A. pleuropneumoniae cells [11, 13]. To date, the most effective strategy relies on targeting a family of toxins centrally involved in A. pleuropneumoniae pathogenesis, the RTX toxins [22, 23]. Vaccines containing RTX toxoids have been shown to effectively prevent the clinical manifestations of the infection [7]. Unfortunately, this approach comes short of stopping lung colonization, and animals immunized in this way have been showed to be possible A. pleuropneumoniae carriers [44]. For this reason we chose to use different combinations of OM proteins and OMVs as immunization strategies, similarly in principle to how the widely used Bexsero ${ }^{\circledR}$ vaccine against Neisseria meningitidis was conceived [45].

Our data showed that none of the immunization strategies employed was able to successfully prevent $A$. pleuropneumoniae colonization or the development of lesions associated with infection. This may be due to different reasons, and our data, together with previously published studies [11, 25, 35], suggest caution in dismissing the selected immunogens as simply ineffective for immunization. As already mentioned, A. pleuropneumoniae possess several resistance mechanisms that allow it to avoid clearance by the host immune system. Accordingly, it is possible that raising antibodies against valid $\mathrm{OM}$ antigenic targets may not be sufficient for the host immune system to clear the infection before the RTX toxins and other virulence factors start to be released. Once released, most of A. pleuropneumoniae toxins can target specifically lymphocytes, and thus kill a wide range of the cells involved in clearance [23]. If this is the case, we hypothesise that a combination of OM immunogens and 

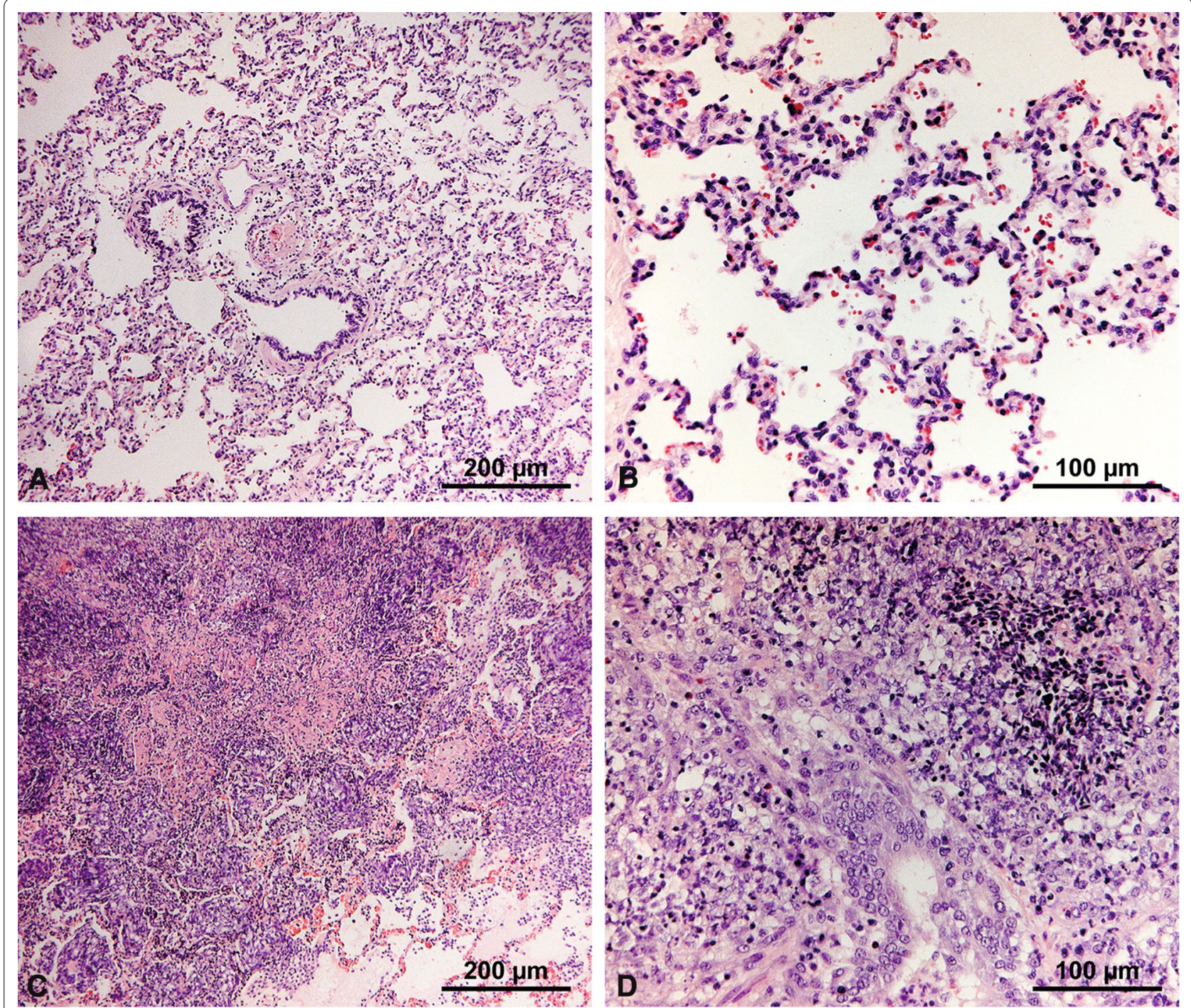

Figure 6 Histological analysis of the lungs. Healthy and affected regions of the lungs were cut out and sectioned for histopathological analysis. Sections were stained using hematoxylin and eosin stain (H\&E). Only selected representative sections are shown for each type of affected animal. A, B Sections of healthy regions of the lungs. Normal structure and cellular components of the lungs are visible. C, D Sections of affected regions of the lungs. Loss of structure and heavy lymphocytic infiltrates are readily appreciable.

RTX toxoids may represent a valid immunization strategy for the prevention of both colonization and pathogenesis by $A$. pleuropneumoniae.

Another possibility comes from the role played by the host immune system during $A$. pleuropneumoniae infections. A. pleuropneumoniae cells are able to induce the release of pro-inflammatory mediators by activated phagocytes during infection, leading to extensive cytotoxicity due to the production of oxygen radicals and proteases [3, 22, 23]. Here we described a correlation between IgG titers against ApfA and VacJ immunogens and lesion scores (Figure 8), suggesting that a different cytotoxic pathway may be involved in the development of the lesions associated with A. pleuropneumoniae. This alternative pathway is known as antibody-dependent cell-mediated cytotoxicity (ADCC), and has been demonstrated to be involved in the pathogenesis of several bacterial infections [46, 47]. Our data point to the involvement of ADCC in A. pleuropneumoniae infections, showing that the groups with the highest IgG titers were the ones that had the highest lesion scores (Figure 8). Furthermore, the histological analysis of the lungs seemed to confirm a major role for the host immune system in the development of the lesions, 


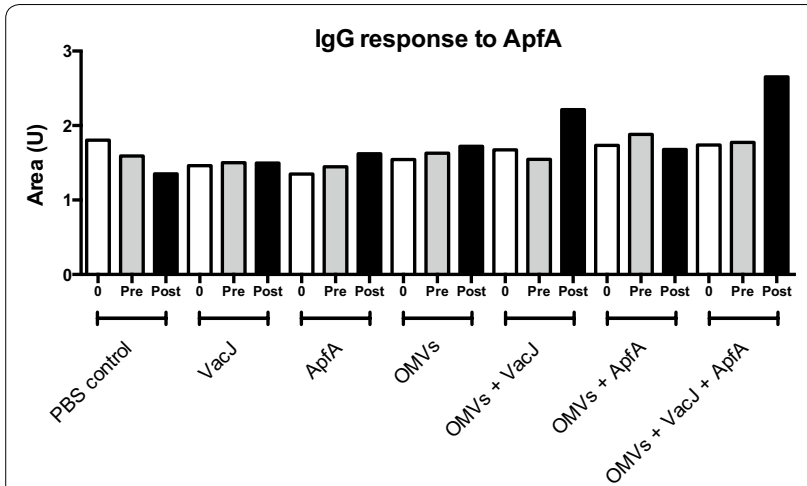

Area Under Curve (AUC)

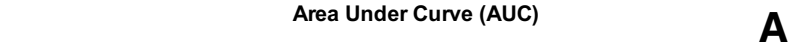

A

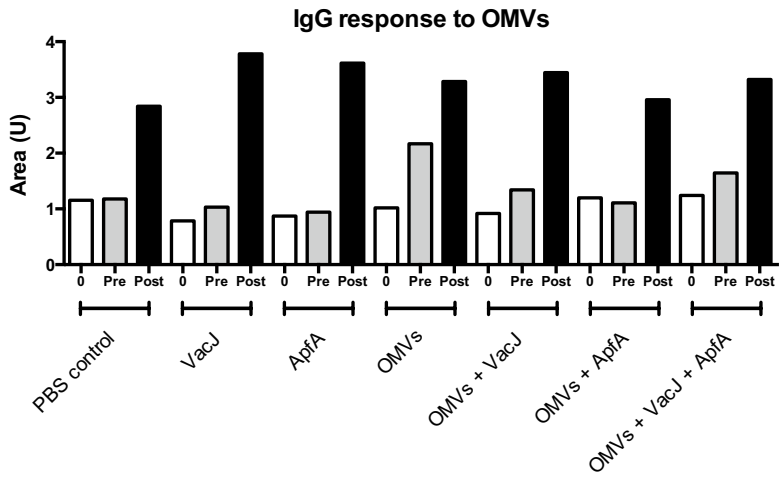

Area Under Curve (AUC)

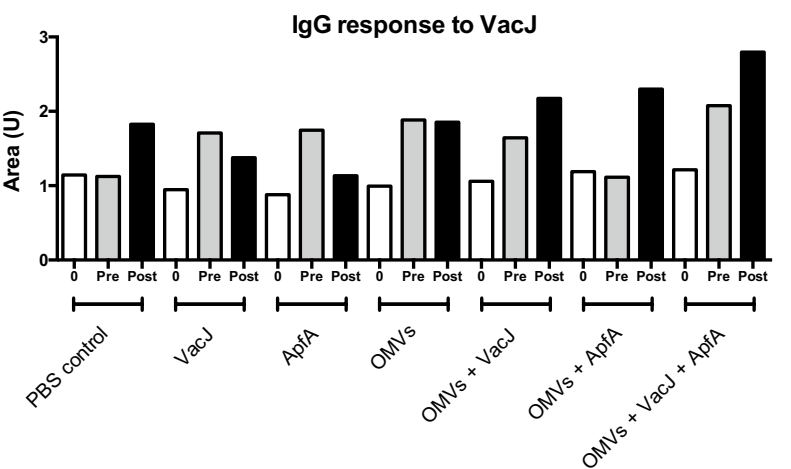

Area Under Curve (AUC)

B

Figure 7 IgG response to the immunogens. Sera from animals within each individual group were pooled together and analysed for lgG concentration against ApfA $(\mathbf{A}), \operatorname{Vac}(\mathbf{B})$ and $\mathrm{OMVs}(\mathbf{C})$. Data are reported as area under curve (AUC), calculated from ELISA titration curves. Three time points are shown: 0: first immunization; pre: second immunization; post: endpoint.
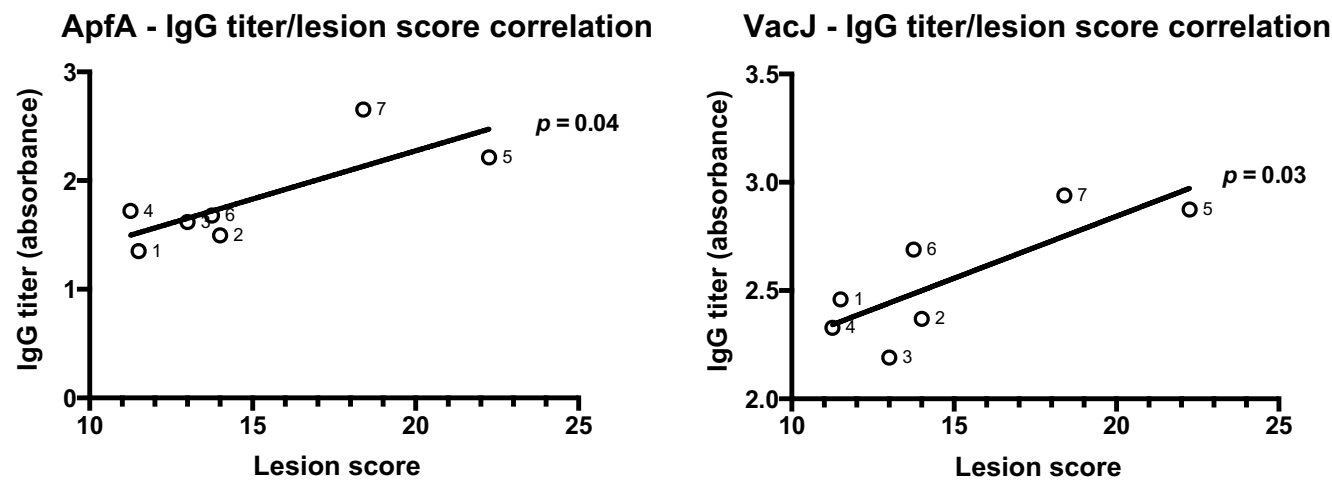

Figure 8 IgG titer/lesion score correlation. Combined lgG titers for each animal group (1-7) against ApfA (A) and VacJ (B) were plotted against the average lesion score of each individual group. Pooled endpoint sera retrieved on the day of euthanasia (1 week after challenge) were used. Statistical analysis by Pearson two-tailed $r$ test showed a significant correlation between average IgG titers and lesion scores (ApfA $P=0.04$; Vac $P=0.03)$.

in which affected lungs were heavily infiltrated by lymphocytes and showing extensive fibrinous exudation on occasions (Figures 4 and 6). This hypothesis may also explain the high variability in lesion score observed between animals belonging to the same group, as the pathological outcome of an A. pleuropneumoniae infection could be much more dependent on the individually variable immune response than on infectious dose (ID). 
Although the role of ADCC in App pathogenicity has yet to be proven, it has been shown that a high seroconversion rate towards the PalA protein produced by App is inversely correlated with protection level in animals immunised with this protein [48]. Notably, no correlation was present between IgG response to the OMVs and lesion scores, likely due to the generalised high response in all animals to the LPS present in the OMVs. Indeed, if ADCC is effectively responsible for considerable damage in A. pleuropneumoniae infections; we could be forced to rethink our approach to both treatment and prevention, as raising IgG titers against $A$. pleuropneumoniae $\mathrm{OM}$ antigens may prove counterproductive. In this scenario, a combination of immunization by RTX toxoids and a desensitisation regime toward ADCC could achieve better results and be instrumental in reducing antibiotic consumption.

As expected, immunization with the OMVs elicited a rather high IgG titer in the animals, exhibiting adjuvant properties when administered in combination with the other immunogens (Figure 7). This did not come as a surprise, as the adjuvant potential of the OMVs has been widely demonstrated in previous studies [29, 49, 50]. On the other hand, ApfA and VacJ elicited a rather low response when administered alone (Figure 7). Our results contrast with previous reports on ApfA, indicating both immunogenic and protective potential in vivo [11,26]. Interestingly, VacJ was found to elicit a consistently high IgG titer after challenge (Figure 7), suggesting that this protein may possess a relevant immunogenic potential in vivo.

Finally, our results show a general reliability of the challenge model used and the effectiveness of the aerosol chamber as a biologically relevant method to study the pathogenesis of $A$. pleuropneumoniae. OMV dosage during immunization was effective in eliciting the maximum immune response within tolerable parameters of toxicity. All animals received approximately the same amount of A. pleuropneumoniae CFU during challenge and developed a subacute infection, while none of them needed to be euthanized before the planned endpoint due to critical clinical conditions. Moreover, the positive correlation between CFU in the lungs and lesion score shows the validity of the scoring system used.

In conclusion, our study provided new insight into $A$. pleuropneumoniae pathogenicity and the role played by host-derived cytotoxicity. The aerosol chamber represented a reliable and effective device for A. pleuropneumoniae challenge, ensuring that all animals were exposed to the same ID through the natural route of infection. On the other hand, the evident contrast between the overall technical reproducibility achieved and the high variability in lesion score within each group underlines the complexity of the A. pleuropneumoniae pathogenesis. To address this issue and provide a more solid statistical basis, larger groups of animals may be needed during similar A. pleuropneumoniae trials in the future.

\section{Abbreviations \\ A. pleuropneumoniae: Actinobacillus pleuropneumoniae; OM: outer membrane; OMV: outer membrane vesicles; NAD: nicotinamide dinucleotide; E. coli: Escherichia coli; PBS: phosphate buffered saline; IM: intramuscular; IN: intra- nasal; BHI: brain-heart infusion; NCTC: National Collection of Type Cultures; $\mathrm{OD}_{600 \mathrm{~nm}}$ : optical density $600 \mathrm{~nm}$; CFU: colony-forming units; ON: overnight; HRP: horseradish peroxidase; ELISA: enzyme-linked immunosorbent assay; ADCC: antibody-dependent cell-mediated cytotoxicity; ID: infectious dose.}

\section{Authors' contributions}

Design of study: AMB, PJH. Immunogens production: FA, CF. Immunization and animal welfare: AMB, PJH, FA, CF. Challenge: AMB, PJH, FA, CF, AR, AD. Postmortem examination: AMB, PJH, FA, CF. Antibody titration: FA. Histopathological analysis of the lungs: CØ. Data analysis: FA. All authors read and approved the final manuscript.

\section{Acknowledgements}

We would like to thank the animal caretakers at the Department of Veterinary and Animal Sciences (University of Copenhagen, Denmark) for the help provided in handling the animals involved in this study.

Competing interests

The authors declare that they have no competing interests.

\section{Ethics approval and consent to participate}

The animal experiments were approved by the Danish National Animal Experiments Inspectorate (Dyreforsøgstilsynet), as stipulated in license number 2014-15-0201-00019.

\section{Funding}

The project was partly funded by The University of Copenhagen, Center for Control of Antimicrobial Resistance (UC-Care), the Center for Research in Pig Production and Health (CPH Pig) at University of Copenhagen, and SEGES Pig Research Center.

\section{Author details}

${ }^{1}$ Department of Veterinary and Animal Sciences, University of Copenhagen, Stigbøjlen 4, 1870 Frb. C., 1-20, Building: 301, Copenhagen, Denmark. ${ }^{2}$ Department of International Health, Immunology and Microbiology ISIM, University of Copenhagen, Øster Farigmagsgade 5, Bldg 22/23, 1014 København K, Copenhagen, Denmark. ${ }^{3}$ Department of Pathology and Pathogen Biology, Royal Veterinary College, Hawkshead Lane, North Mymms, Hertfordshire AL9 7TA, UK. ${ }^{4}$ Department of Biomedical Sciences, University of Copenhagen, Blegdamsvej 3, 2200 København N, 12.3, Building: 32, Copenhagen, Denmark.

\section{Publisher's Note}

Springer Nature remains neutral with regard to jurisdictional claims in published maps and institutional affiliations.

Received: 25 October 2017 Accepted: 18 December 2017

Published online: 09 January 2018

\section{References}

1. Losinger WC (2005) Economic impacts of reduced pork production associated with the diagnosis of Actinobacillus pleuropneumoniae on grower/ finisher swine operations in the United States. Prev Vet Med 68:181-193

2. PROHEALTH economic impact of Actinobacillus pleuropneumoniae. http:// www.fp7-prohealth.eu/news-index/newsletter-november-2015/production-diseases-cost-pig-producers/. Accessed 4 Apr 2017 
3. Bossé JT, Janson H, Sheehan BJ, Beddek AJ, Rycroft AN, Kroll JS, Langford PR (2002) Actinobacillus pleuropneumoniae: pathobiology and pathogenesis of infection. Microbes Infect 4:225-235

4. Bossé JT, Li Y, Sárközi R, Gottschalk M, Angen $\varnothing$, Nedbalcova K, Rycroft AN, Fodor L, Langford PR (2017) A unique capsule locus in the newly designated Actinobacillus pleuropneumoniae serovar 16 and development of a diagnostic PCR assay. J Clin Microbiol 55:902-907

5. Sarkozi R, Makrai L, Fodor L (2015) Identification of a proposed new serovar of Actinobacillus Pleuropneumoniae: serovar 16. Acta Vet Hung 63:444-450

6. Negrete-Abascal E, Tenorio VR, Guerrero AL, García RM, Reyes ME, de la Garza M (1998) Purification and characterization of a protease from Actinobacillus pleuropneumoniae serotype 1, an antigen common to all the serotypes. Can J Vet Res 62:183-190

7. Ramjeet M, Deslandes V, Gouré J, Jacques M (2008) Actinobacillus pleuropneumoniae vaccines: from bacterins to new insights into vaccination strategies. Anim Health Res Rev 9:25-45

8. Hensel A, Huter V, Katinger A, Raza P, Strnistschie C, Roesler U, Brand E, Lubitz W (2000) Intramuscular immunization with genetically inactivated (ghosts) Actinobacillus pleuropneumoniae serotype 9 protects pigs against homologous aerosol challenge and prevents carrier state. Vaccine 18:2945-2955

9. Reimer D, Frey J, Jansen R, Veit HP, Inzana TJ (1995) Molecular investigation of the role of Apxl and Apxll in the virulence of Actinobacillus pleuropneumoniae serotype 5. Microb Pathog 18:197-209

10. Chiers K, van Overbeke I, De Laender P, Ducatelle R, Carel S, Haesebrouck F (1998) Effects of endobronchial challenge with Actinobacillus pleuropneumoniae serotype 9 of pigs vaccinated with inactivated vaccines containing the Apx toxins. Vet Q 20:65-69

11. Sadilkova L, Nepereny J, Vrzal V, Sebo P, Osicka R (2012) Type IV fimbrial subunit protein ApfA contributes to protection against porcine pleuropneumonia. Vet Res 43:2

12. Del Pozo Sacristán R, Michiels A, Martens M, Haesebrouck F, Maes D (2014) Efficacy of vaccination against Actinobacillus pleuropneumoniae in two Belgian farrow-to-finish pig herds with a history of chronic pleurisy. Vet Rec 174:302-302

13. Tumamao JQ, Bowles RE, van den Bosch H, Klaasen HLBM, Fenwick BW, Storie GJ, Blackall PJ (2004) Comparison of the efficacy of a subunit and a live streptomycin-dependent porcine pleuropneumonia vaccine. Aust Vet J 82:370-374

14. Aarestrup FM, Duran OC, Burch DGS (2008) Antimicrobial resistance in swine production. Anim Heal Res Rev 9:135-148

15. Vanni M, Merenda M, Barigazzi G, Garbarino C, Luppi A, Tognetti R, Intorre $L$ (2012) Antimicrobial resistance of Actinobacillus pleuropneumoniae isolated from swine. Vet Microbiol 156:172-177

16. Kim B, Hur J, Lee JY, Choi Y, Lee JH (2016) Molecular serotyping and antimicrobial resistance profiles of Actinobacillus pleuropneumoniae isolated from pigs in South Korea. Vet Q 36:137-144

17. Huang H, Potter AA, Campos M, Leighton FA, Willson PJ, Yates WD (1998) Pathogenesis of porcine Actinobacillus pleuropneumonia: part I. Effects of surface components of Actinobacillus pleuropneumoniae in vitro and in vivo. Can J Vet Res 62:93-101

18. Huang H, Potter AA, Campos M, Leighton FA, Willson PJ, Haines DM, Yates WD (1999) Pathogenesis of porcine Actinobacillus pleuropneumonia, part II: roles of proinflammatory cytokines. Can J Vet Res 63:69-78

19. Chiers K, De Waele T, Pasmans F, Ducatelle R, Haesebrouck F (2010) Virulence factors of Actinobacillus pleuropneumoniae involved in colonization, persistence and induction of lesions in its porcine host. Vet Res 41:65

20. Klitgaard K, Friis C, Jensen TK, Angen $\varnothing$, Boye M (2012) Transcriptional portrait of Actinobacillus pleuropneumoniae during acute disease-potential strategies for survival and persistence in the host. PLoS One 7:e35549

21. González OG, García RM, De La Garza M, Vaca PS, Paniagua GL, Mejía R, Tenorio VR, Negrete-Abascal E (2004) Actinobacillus pleuropneumoniae metalloprotease: cloning and in vivo expression. FEMS Microbiol Lett 234:81-86

22. Frey J (1995) Virulence in Actinobacillus pleuropneumoniae and RTX toxins. Trends Microbiol 3:257-260

23. Frey J (2011) The role of RTX toxins in host specificity of animal pathogenic Pasteurellaceae. Vet Microbiol 153:51-58

24. Hoeltig D, Hennig-Pauka I, Thies K, Rehm T, Beyerbach M, StrutzbergMinder K, Gerlach GF, Waldmann KH (2009) A novel Respiratory Health
Score (RHS) supports a role of acute lung damage and pig breed in the course of an Actinobacillus pleuropneumoniae infection. BMC Vet Res 5:14

25. Antenucci F, Fougeroux C, Bossé JT, Magnowska Z, Roesch C, Langford P, Holst PJ, Bojesen AM (2017) Identification and characterization of serovarindependent immunogens in Actinobacillus pleuropneumoniae. Vet Res 48:74

26. Zhou Y, Li L, Chen Z, Yuan H, Chen H, Zhou R (2013) Adhesion protein ApfA of Actinobacillus pleuropneumoniae is required for pathogenesis and is a potential target for vaccine development. Clin Vaccine Immunol 20:287-294

27. Xie F, Li G, Zhang W, Zhang Y, Zhou L, Liu S, Liu S, Wang C (2016) Outer membrane lipoprotein VacJ is required for the membrane integrity, serum resistance and biofilm formation of Actinobacillus pleuropneumoniae. Vet Microbiol 183:1-8

28. Kulp A, Kuehn MJ (2012) Biological functions and biogenesis of secreted bacterial outer membrane vesicles. Annu Rev Microbiol 64:163-184

29. Collins BS (2011) Gram-negative outer membrane vesicles in vaccine development. Discov Med 12:7-15

30. van der Pol L, Stork M, van der Ley P (2015) Outer membrane vesicles as platform vaccine technology. Biotechnol J 10:1689-1706

31. Negrete-Abascal E, García RM, Reyes ME, Godínez D, De La Garza M (2000) Membrane vesicles released by Actinobacillus pleuropneumoniae contain proteases and Apx toxins. FEMS Microbiol Lett 191:109-113

32. Chen DJ, Osterrieder N, Metzger SM, Buckles E, Doody AM, DeLisa MP, Putnam D (2010) Delivery of foreign antigens by engineered outer membrane vesicle vaccines. Proc Natl Acad Sci U S A 107:3099-3104

33. Sanders H, Feavers IM (2011) Adjuvant properties of meningococcal outer membrane vesicles and the use of adjuvants in Neisseria meningitidis protein vaccines. Expert Rev Vaccines 10:323-334

34. Pritsch M, Ben-Khaled N, Chaloupka M, Kobold S, Berens-Riha N, Peter A, Liegl G, Schubert S, Hoelscher M, Löscher T, Wieser A (2016) Comparison of intranasal outer membrane vesicles with cholera toxin and injected MF59C.1 as adjuvants for malaria transmission blocking antigens AnAPN1 and Pfs48/45. J Immunol Res 2016:3576028

35. Pors SE, Pedersen IJ, Skjerning RB, Thøfner ICN, Persson G, Bojesen AM (2016) Outer membrane vesicles of Gallibacterium anatis induce protective immunity in egg-laying hens. Vet Microbiol 195:123-127

36. Torremorell M, Pijoan C, Janni K, Walker R, Joo HS (1997) Airborne transmission of Actinobacillus pleuropneumoniae and porcine reproductive and respiratory syndrome virus in nursery pigs. Am J Vet Res 58:828-832

37. Jobert JL, Savoye C, Cariolet R, Kobisch M, Madec F (2000) Experimental aerosol transmission of Actinobacillus pleuropneumoniae to pigs. Can J Vet Res 64:21-26

38. Jacobsen MJ, Nielsen JP, Nielsen R (1996) Comparison of virulence of different Actinobacillus pleuropneumoniae serotypes and biotypes using an aerosol infection model. Vet Microbiol 49:159-168

39. Maas A, Jacobsen ID, Meens J, Gerlach GF (2006) Use of an Actinobacillus pleuropneumoniae multiple mutant as a vaccine that allows differentiation of vaccinated and infected animals. Infect Immun 74:4124-4132

40. Menzel A, Beyerbach M, Siewert C, Gundlach M, Hoeltig D, Graage R, Seifert H, Waldmann KH, Verspohl J, Hennig-Pauka I (2014) Actinobacillus pleuropneumoniae challenge in swine: diagnostic of lung alterations by infrared thermography. BMC Vet Res 10:199

41. Thrane S, Janitzek CM, Agerbæk M $\varnothing$, Ditlev SB, Resende M, Nielsen MA, Theander TG, Salanti A, Sander AF (2015) A novel virus-like particle based vaccine platform displaying the placental malaria antigen VAR2CSA. PLoS One 10:e0143071

42. Hannan PC, Bhogal BS, Fish JP (1982) Tylosin tartrate and tiamutilin effects on experimental piglet pneumonia induced with pneumonic pig lung homogenate containing mycoplasmas, bacteria and viruses. Res Vet Sci 33:76-88

43. Shao M, Wang Y, Wang C, Guo Y, Peng Y, Liu J, Li G, Liu H, Liu S (2010) Evaluation of multicomponent recombinant vaccines against ActinobacilI us pleuropneumoniae in mice. Acta Vet Scand 52:52

44. Van Overbeke I, Chiers K, Ducatelle R, Haesebrouck F (2001) Effect of endobronchial challenge with Actinobacillus pleuropneumoniae serotype 9 of pigs vaccinated with a vaccine containing APX toxins and transferrinbinding proteins. J Vet Med B Infect Dis Vet Public Health 48:15-20

45. Vernikos G, Medini D (2014) Bexsero ${ }^{\circledR}$ chronicle. Pathog Glob Health 108:305-316 
46. Hagberg M, Ahlstedt S, Hanson L (1982) Antibody-dependent cell-mediated cytotoxicity against Escherichia coli $\mathrm{O}$ antigens. Eur J Clin Microbiol 1:59-65

47. Xie X, McLean MD, Hall JC (2010) Antibody-dependent cell-mediated cytotoxicity- and complement-dependent cytotoxicity-independent bactericidal activity of an IgG against Pseudomonas aeruginosa O6ad. J Immunol 184:3725-3733

48. van den Bosch H, Frey J (2003) Interference of outer membrane protein PalA with protective immunity against Actinobacillus pleuropneumoniae infections in vaccinated pigs. Vaccine 21:3601-3607
49. Price NL, Goyette-Desjardins G, Nothaft H, Valguarnera E, Szymanski CM, Segura M, Feldman MF (2016) Glycoengineered outer membrane vesicles: a novel platform for bacterial vaccines. Sci Rep 6:24931

50. Acevedo R, Fernández S, Zayas C, Acosta A, Sarmiento ME, Ferro VA, Rosenqvist E, Campa C, Cardoso D, Garcia L, Perez JL (2014) Bacterial outer membrane vesicles and vaccine applications. Front Immunol 5:121

\section{Submit your next manuscript to BioMed Central and we will help you at every step:}

- We accept pre-submission inquiries

- Our selector tool helps you to find the most relevant journal

- We provide round the clock customer support

- Convenient online submission

- Thorough peer review

- Inclusion in PubMed and all major indexing services

- Maximum visibility for your research

Submit your manuscript at

www.biomedcentral com/submit 